\title{
Spectrophotometric Methods for the Assay of Ceftazidime in Bulk and its Pharmaceutical Formulations
}

\author{
L. MOHAN KRISHNA ${ }^{\mathrm{a}^{*}}$, P. JAYACHANDRA REDDY ${ }^{\mathrm{a}}$, \\ V. JAYA SANKAR REDDY ${ }^{\mathrm{a}}$ and K.V. S. PRASADA RAO ${ }^{\mathrm{b}}$ \\ ${ }^{a}$ Krishna Teja Pharmacy College, Tirupati, India \\ ${ }^{\mathrm{b}}$ Rahul Institute of Pharmaceutical Sciences, Chirla, India \\ mohanlokireddy@gmail.com
}

Received 1 November 2012 / Accepted 12 December 2012

\begin{abstract}
Three visible spectrophotometric methods have been described for the assay of ceftazidime (CTZ) either in bulk form or dosage forms. Methods A-C are based on the oxidation of CTZ with an excess of oxidant ( $N$-bromosuccinimide (NBS) in methods A and B or chloramine-T (CAT) in method $\mathrm{C}$ ) in acidic medium. The unreacted oxidant was then estimated colorimetrically by using an oxidisable dye (Celistine blue (CB) in method A or gallocyanine (GC) in method C) or by $p$ - $N$-methyl aminophenol sulphate (PMAP)- sulphanilamide (SA) reagent in method B. Regression analysis of Beer's law plots showed good concentration range $2.0-10.0 \mu \mathrm{g} / \mathrm{mL}, 4.0-16.0 \mu \mathrm{g} / \mathrm{mL}$ and $1.0-8.0 \mu \mathrm{g} / \mathrm{mL}$ for methods A, B and C respectively and gives reproducible results.
\end{abstract}

Keywords: Spectrophotometry, NBS, Ceftazidime, Gallocyanine

\section{Introduction}

Ceftazidime (CTZ) is a thrid-generation cephalosporin antibiotic. Like other thirdgeneration cephalosporins, it has broad spectrum activity against gram-positive and garmnegative bacteria. Unlike most third-generation agents, it is active against pseudomonas aeruginosa, however it has weaker activity against gram-positive microorganisms and it is not used for such infections. It is also used in the empirical therapy of febrile neutropenia, in combination with other antibiotics, chemically known as (6R,7R,a)-7-(2-aminothiazol4-yl)- 2-(2-carboxypropan-2-yloxyimino) acetamindo)-8-oxo-3-(pyridinium-1-ylmethyl)5thia-1-aza-bicyclo [4.2.0] oct-2-ene-2-carboxylate. A number of methods such as Spectrophotometic ${ }^{1-12}$ and HPLC ${ }^{13-29}$, were reported for the estimation of CTZ. Literature survey revealed that only two visible spectrophotometric methods were reported for it quantitative determination in bulk drug and pharmaceutical formulations. The present communication describes three visible spectrophotometric methods (A-C) for the assay of CTZ in bulk form and dosage forms. Methods A-C are indirect procedures, involving the addition of an excess oxidant and determination of the unreacted oxidant by measuring 
either the decrease in absorbance of the dye (NBS/CB ${ }^{9}$, method A; CAT/GC ${ }^{11}$, method C) or color produced with PMAP-SA reagent (NBS/PMAP-SA ${ }^{10}$, method B).

\section{Experimental}

A Milton Roy Spectronic 1201 with $1 \mathrm{~cm}$ matched quartz cells were used for the spectral and absorbance measurements. An Elico LI-120 digital $\mathrm{pH}$ meter was used for $\mathrm{pH}$ measurements.

\section{Reagents}

All the chemicals and reagents were of analytical grade and the solutions were prepared in triply distilled water. Aqueous solutions of NBS (Loba, $5.62 \times 10^{-4} \mathrm{M}$ ), CB (Chroma, $5.49 \times 10^{-4} \mathrm{M}$ ) and $\mathrm{HCl}$ (E-merck, 5M) were prepared for method A. Aqueous solutions of NBS (Loba, $5.62 \times 10^{-3} \mathrm{M}$ ), PMAP (Loba, 8.71 $\times 10^{-3} \mathrm{M}$ ), SA (Sd fine chemicals, $1.16 \times 10^{-2} \mathrm{M}$ ) and acetic acid (Qualigens, $8.75 \times 10^{-1} \mathrm{M}$ ) were prepared for method $\mathrm{B}$. Aqueous solutions of CAT (Loba, $7.10 \times 10^{-4} \mathrm{M}$ ) and GC (Chroma, $2.9 \times 10^{-4} \mathrm{M}$ ) were prepared for method C.

\section{Preparation of standard drug solution}

One $\mathrm{mg} / \mathrm{mL}$ solution was prepared by dissolving $100 \mathrm{mg}$ of pure CTZ in $100 \mathrm{~mL}$ of distilled water and this stock solution was diluted stepwise with distilled water to obtain the working standard solution of concentrations $100 \mu \mathrm{g} / \mathrm{mL}$ for method A, $200 \mu \mathrm{g} / \mathrm{mL}$ for method B and $50 \mu \mathrm{g} / \mathrm{mL}$ for methods $\mathrm{C}$ respectively.

\section{Analytical procedures}

\section{Method A}

Aliquots of standard CTZ solution (1.0-3.0 mL, $50 \mu \mathrm{g} / \mathrm{mL}), 1.25 \mathrm{~mL}$ of $5 \mathrm{M} \mathrm{HCl}$ and $2.5 \mathrm{~mL}$ of NBS $\left(5.62 \times 10^{-4} \mathrm{M}\right)$ were delivered into a series of $25 \mathrm{~mL}$ calibrated tubes and the volume in each tube was brought to $20 \mathrm{~mL}$ with distilled water. After $10 \mathrm{~min}, 5 \mathrm{~mL}$ of CB solution was added and mixed thoroughly. The absorbances were measured after 5 min at $520 \mathrm{~nm}$ against distilled water. The blank (omitting drug) and dye (omitting drug and oxidant) solutions were prepared in a similar manner and their absorbances were measured against distilled water. The difference in the decrease in absorbance between test and blank (or test against reagent blank) corresponding to the consumed NBS and in turn drug concentration was computed from its calibration graph.

\section{Method B}

Aliquots of standard CTZ solution $(1.0-3.0 \mathrm{~mL}, 100 \mu \mathrm{g} / \mathrm{mL})$ were transferred into a series of $25 \mathrm{~mL}$ calibrated tubes. $0.5 \mathrm{~mL}$ of $\mathrm{AcOH}$ and $2.0 \mathrm{~mL}$ of $\mathrm{NBS}\left(5.62 \times 10^{-3} \mathrm{M}\right)$ solutions were added to the above solutions and volume in each tube was brought to $10 \mathrm{~mL}$ with distilled water and kept aside for $20 \mathrm{~min}$ at room temp. Then $2.0 \mathrm{~mL}$ of PMAP solution was added. After $2 \mathrm{~min}, 2.0 \mathrm{~mL}$ of SA solution was added and the volume was made up to the mark with distilled water. The absorbances were measured after 10 min at $520 \mathrm{~nm}$ against distilled water. A blank experiment was also carried out omitting the drug. The decrease in the absorbance and in turn the drug concentration was obtained by subtracting the absorbance of the test solution from the blank. The amount of CTZ was computed from its calibration graph. 


\section{Method C}

To each of $25 \mathrm{~mL}$ calibrated tubes containing standard CTZ solution $(1.0-3.0 \mathrm{~mL}, 50 \mu \mathrm{g} / \mathrm{mL}$ ), $1.25 \mathrm{~mL}$ of $5 \mathrm{M} \mathrm{HCl}$ and $2.0 \mathrm{~mL}$ of CAT were added and the solution was diluted to $20 \mathrm{~mL}$ with distilled water. After $10 \mathrm{~min}, 5 \mathrm{~mL}$ of GC solution was added, mixed thoroughly and the absorbances were measured after $15 \mathrm{~min}$ at $540 \mathrm{~nm}$ against distilled water. A blank was carried out in a similar manner. The decrease in absorbance corresponding to consumed CAT, which in turn to the drug quantity was obtained by subtracting the absorbance of the blank solution from that of the test solution. The calibration graph was drawn by plotting the decrease in the absorbance of the dye (GC), against amount of the drug. Amount of the drug in any sample was computed from its calibration graph.

\section{For pharmaceutical formulations}

The injection powder equivalent to $100 \mathrm{mg}$ of CTZ was accurately weighed and dissolved in $100 \mathrm{~mL}$ of distilled water to achieve a drug concentration of $1 \mathrm{mg} / \mathrm{mL}$. From which suitable dilutions were performed for methods $\mathrm{A}, \mathrm{B}$ and $\mathrm{C}$ as mentioned above.

\section{Results and Discussion}

The optimum conditions for the color development of the method were established by varying the parameters one at a time in each method, keeping the others fixed and observing the effect produced on the absorbance of the colored species.

Method A involves the oxidation of CTZ with excess of NBS (first step) and estimating the unreacted NBS with CB (second step). The effect of reagent concentration (acidity, NBS and $\mathrm{CB}$ ) and time in each step were studied by means of controlled experiments varying one perameter at a time. Studies of variation of acid concentration indicated that constant absorbance was obtained with 1.0-1.5 mL of $5 \mathrm{M} \mathrm{HCl}$, 0.5-1.0 $\mathrm{mL}$ of $5 \mathrm{M} \mathrm{H}_{2} \mathrm{SO}_{4}$, or 1.5-2.5 mL of $5 \mathrm{M} \mathrm{AcOH}$ when $3.0 \mathrm{~mL}$ of $5.62 \times 10^{-4} \mathrm{M}$ NBS was used. Since the difference in absorbance between the sample and blank was found to be highest with the addition of $\mathrm{HCl}$ and subsequent studies were performed with $1.25 \mathrm{~mL}$ of $5 \mathrm{M} \mathrm{HCl}$. In order to ascertain the linear relationship between the volume of added NBS and decrease in absorbance of CB, experiments were carried out in $1.25 \mathrm{~mL}$ of $5 \mathrm{M} \mathrm{HCl}$ with varying volumes of NBS. The decrease in absorbance was found to be linear up to $2.5 \mathrm{~mL}$ of $5.62 \times 10^{-4} \mathrm{M}$ NBS with $5.0 \mathrm{~mL}$ of $5.49 \times 10^{-4} \mathrm{M}$ CB. So fixed amounts of $\mathrm{HCl}(1.25 \mathrm{~mL}, 5 \mathrm{M})$, NBS $\left(2.5 \mathrm{~mL}, 5.618 \times 10^{-4} \mathrm{M}\right)$, $\mathrm{CB}\left(5.0 \mathrm{~mL}, 5.49 \times 10^{-4} \mathrm{M}\right)$ were taken for further investigation. Time span of 5 to $15 \mathrm{~min}$ for the reaction between GAT and NBS in the first step and 3 to 10 min between NBS and CB in the second step resulted in constant and maximum difference in absorbance of test and blank solutions. Hence reaction periods of 10 and 5 min were maintained in subsequence studies of the first and second step respectively. The color was found to be stable up to $30 \mathrm{~min}$. The absorption spectra of the colored species in the proposed method show characteristic $\lambda_{\max } 520 \mathrm{~nm}$.

Method B involves two stages, namely oxidation with excess NBS and the determination of unreacted NBS using PMAP-SA reagent. Oxidation of CTZ with 1.5-3.0 mL of NBS $\left(5.61 \times 10^{-3} \mathrm{M}\right)$ solution gave maximum and reproducible absorbance values. The effect of time and temperature of oxidation on the absorbance of the colored species was studied by conducting the oxidation at different temperatures for different time intervals. Oxidation times ranging from $10-20 \mathrm{~min}$ at room temp $\left(28 \pm 5^{\circ} \mathrm{C}\right)$ gave constant and reproducible absorbance values. Prolonging the oxidation time beyond $20 \mathrm{~min}$ and increasing the temperature gave erratic results. Maintaining the $\mathrm{pH}$ of the solution at $2.9 \pm 0.2$ 
was found to be the best for attaining maximum sensitivity. This was achieved by the addition of $0.5 \mathrm{~mL}$ of $8.75 \times 10^{-1} \mathrm{M}$ acetic acid. Use of $1.0-2.0 \mathrm{~mL}$ of PMAP solution and 1.0-2.5 mL of SA solution afforded maximum absorbance value. A waiting period of 1-3 min was necessary between the addition of PMAP and SA solutions for the generation of $p$ - $N$-methyl benzoquinone monoimine (PMBQMI) by the action of NBS on PMAP. Prolonging the waiting period beyond 3 min resulted in low absorbance values, owing to the partial hydrolysis of PMBQMI formed in situ to the quinone state. Among the water miscible solvents examined, water was found to be the best for final dilution of the solution. Maximum color intensity was attained within $10 \mathrm{~min}$ after the final dilution and remained stable for next $40 \mathrm{~min}$. The absorption spectra of the colored species in the proposed method show characteristic $\lambda_{\max } 520 \mathrm{~nm}$.

Method C involves the oxidation of CTZ with excess of CAT (first step) and estimating the unreacted CAT with GC (second step). The effect of reagent concentration (acidity, CAT and GC) and time in each step was studied by means of controlled experiments by varying one parameter at a time. Studies of variation of acid concentration indicated that constant absorbance was obtained with 1.0-1.5 mL of $5 \mathrm{M} \mathrm{HCl}, 0.5-1.0 \mathrm{~mL}$ of $5 \mathrm{M} \mathrm{H}_{2} \mathrm{SO}_{4}$, or $1.5-2.5 \mathrm{~mL}$ of $5 \mathrm{M} \mathrm{AcOH}$ when $3.0 \mathrm{~mL}$ of CAT was used. Since the difference in absorbance between the sample and blank was found to be highest with the addition of $\mathrm{HCl}$ and subsequent studies were performed with $1.25 \mathrm{~mL}$ of $5 \mathrm{M} \mathrm{HCl}$. In order to ascertain the linear relationship between the volume of added CAT and decrease in absorbance of GC, experiments were carried out in $1.25 \mathrm{~mL}$ of $5 \mathrm{M} \mathrm{HCl}$ with varying volumes of CAT. The decrease in absorbance was found to be linear up to $2.0 \mathrm{~mL}$ of CAT with $5.0 \mathrm{~mL}$ of GC. So fixed amounts of $\mathrm{HCl}(1.25 \mathrm{~mL}, 5 \mathrm{M})$, CAT $\left(2.0 \mathrm{~mL}, 7.10 \times 10^{-4} \mathrm{M}\right)$, GC $\left(5.0 \mathrm{~mL}, 2.9 \times 10^{-4} \mathrm{M}\right)$ were taken for further investigation. Time span of 5 to $15 \mathrm{~min}$ for the reaction between CTZ and CAT in the first step and 10 to 20 min between CAT and GC in the second step resulted in constant and maximum difference in absorbance of test and bulk solutions. Hence reaction periods of 10 and 15 min were maintained in subsequence studies of the first and second step respectively. The color was found to be stable up to $60 \mathrm{~min}$. The absorption spectra of the colored species in the proposed method show characteristic $\lambda_{\max } 540 \mathrm{~nm}$.

\section{Analytical data}

The optical characteristics such as Beer's law limits, molar absorptivity for each method are given in Table 1. The precision of each method was found by measuring absorbances of six replicate samples containing known amounts of drug and the results obtained are incorporated in Table 1. Regression analysis using the method of least squares was made to evaluate the slope (b), intercept (a) and correlation coefficient (r) for each method and are presented in Table 1. Commercial formulations containing GAT were successfully analyzed by the proposed methods. The results obtained by the proposed and reference methods (UV) for dosage forms were compared statistically by the $t$ - and $F$ - tests (Table 2). This comparison shows that there is no significant difference between the results of proposed methods and those of the reference ones. The similarity of the results is obvious evidence that during the application of these methods, the additives and excipients that are usually present in tablets do not interfere in the assay of proposed methods. As an additional check of accuracy of the proposed methods, recovery experiments were performed by adding a fixed amount of the drug to the pre-analysed formulations. The amount of drug found, the $\%$ recovery was calculated in the usual way. 
Table 1. Optical characteristic, precision and accuracy of the proposed methods for CFT

\begin{tabular}{lccc}
\hline \multirow{2}{*}{ Optical characteristics } & Method A & Method B & Method C \\
\cline { 2 - 4 } & NBS/CB & NBS/PMAP-SA & CAT/GC \\
\hline$\lambda_{\max }, \mathrm{nm}$ & 520 & 520 & 540 \\
Beer's Law limits, $\mu \mathrm{g} / \mathrm{mL}$ & $2.0-10.0$ & $4.0-20.0$ & $1.0-6.0$ \\
Molar absorptivity, $\mathrm{L} \mathrm{mol}^{-1} \mathrm{~cm}^{-1}$ & $4.431 \times 10^{4}$ & $3.227 \times 10^{4}$ & $4.787 \times 10^{4}$ \\
Sandell's sensitivity & 0.014 & 0.020 & 0.013 \\
$\left(\mu \mathrm{g} / \mathrm{cm}^{2} / 0.001\right.$ absorbance unit) & & & \\
Regression Equation y = a + bc & 0.0692 & 0.0632 & 0.0753 \\
(i) Slope (b) & 0.0014 & -0.127 & 0.0012 \\
(ii) Intercept (a) & 0.9998 & 0.9999 & 0.9998 \\
Correlation coefficient (r) & 0.359 & 0.074 & -0.133 \\
\% Error in bulk sample & 0.362 & 0.317 & 0.336 \\
\% Range of error 0.05 level & 0.531 & 0.469 & 0.497 \\
0.01 Level & 0.4291 & 0.3795 & 0.4016 \\
Relative standard deviation & ${ }^{*}$ & &
\end{tabular}

${ }^{*}$ Average of six determinations considered. ${ }^{* *}$ Average of three determinations

Table 2. Assay of CFT in pharmaceutical formulations

\begin{tabular}{|c|c|c|c|c|c|c|c|}
\hline \multirow{2}{*}{$\begin{array}{c}\text { Pharmaceutical formulations } \\
\text { (Labelled amount) }\end{array}$} & \multicolumn{3}{|c|}{ Amount found by proposed methods ${ }^{*}$} & \multirow{2}{*}{$\begin{array}{l}\text { Reference } \\
\text { method }\end{array}$} & \multicolumn{3}{|c|}{ \% Recovery by proposed methods ${ }^{* *}$} \\
\hline & A & B & $\mathrm{C}$ & & A & B & $\mathrm{C}$ \\
\hline Inj.I & $199.6 \pm 0.38$ & $199.78 \pm 0.38$ & $199.55 \pm 0.42$ & $199.62 \pm 0.33$ & $99.79 \pm 0.19$ & $99.89 \pm 0.20$ & $99.77 \pm 0.21$ \\
\hline (200 mg) & $F=1.35, t=0.61$ & $\mathrm{~F}=1.34, \mathrm{t}=0.98$ & $\mathrm{~F}=1.64, \mathrm{t}=0.6$ & & & & \\
\hline Inj.II & $199.74 \pm 0.44$ & $199.12 \pm 1.0$ & $199.6 \pm 0.6$ & $199.34 \pm 0.71$ & $99.87 \pm 0.22$ & $99.56 \pm 0.5$ & $99.44 \pm 0.64$ \\
\hline (200 mg) & $\mathrm{F}=2.66, \mathrm{t}=1.22$ & $\mathrm{~F}=1.95, \mathrm{t}=1.3$ & $\mathrm{~F}=1.46, \mathrm{t}=1.4$ & & & & \\
\hline Inj.III & $398.42 \pm 1.43$ & $399.83 \pm 2.59$ & $399.68 \pm 2.86$ & $396.55 \pm 1.94$ & $99.60 \pm 0.35$ & $99.95 \pm 0.64$ & $99.92 \pm 0.71$ \\
\hline (400 mg) & $\mathrm{F}=1.83, \mathrm{t}=1.99$ & $\mathrm{~F}=1.00, \mathrm{t}=0.83$ & $\mathrm{~F}=1.10, \mathrm{t}=0.99$ & & & & \\
\hline Inj. IV & $398.56 \pm 1.43$ & $396.79 \pm 2.4$ & $398.85 \pm 1.94$ & $397.33 \pm 2.58$ & $99.64 \pm 0.35$ & $99.19 \pm 0.60$ & $99.72 \pm 0.48$ \\
\hline (400 mg) & $\mathrm{F}=1.83, \mathrm{t}=-5.00$ & $\mathrm{~F}=1.15, \mathrm{t}=1.19$ & $\mathrm{~F}=2.41, \mathrm{t}=0.18$ & & & & \\
\hline
\end{tabular}

${ }^{\#}$ Developed in the laboratory using methanol,${ }^{*}$ Average \pm standard deviation of six determinations; the $t$ - and $F$ - values refer to comparison of the proposed method with the reference method. Theoretical values at $95 \%$ confidence limit, $t=2.57, F=5.05 .{ }^{* *}$ After adding 3 different amounts of the pure labeled to the pharmaceutical formulation, each value is an average of 3 determinations 


\section{Interference studies}

The interference studies in the determination of CTZ in pharmaceutical formulation revealed that the normally existing excipients and additives like starch, lactose, gelatin, talc, magnesium stearate, aluminum hydroxide, sorbitol, calcium silicate and glycerin do not interfere even when present in excess than the anticipated amount. However, a preliminary clean up procedure with methanol is necessary to avoid interference due to the presence of reducing sugars like lactose if present, prior to the estimation of CTZ in formulations for methods A, B and C respectively.

\section{Chemistry of colored species}

\section{Methods $A$ and $B$}

These methods are based on the oxidation of CTZ by NBS to form oxidation products (probably mixtures, but reproducible under proposed experimental conditions) besides unreacted NBS, followed by the estimation of unreacted NBS either by CB (method A) or PMAP-SA (method B). In method A, the unreacted NBS decreases the color intensity of CB. NBS is involved in bromination reaction with the dye to form a brominated dye which is colorless. The probable sequences of reactions through analogy are presented in scheme A. In method B, the unreacted NBS develops color when treated with PMAP-SA. The PMBQMI formed in situ from PMAP and NBS involves charge-transfer color complex formation with SA.

\section{Method C}

In method C, CAT undergoes hydrolysis in aqueous acid medium to give sodium hypochlorite followed by hypochlorous acid. This reacts with CTZ to form the relevant oxidation products, probably a mixture, which appears to be reproducible under the specified experimental conditions. The remaining hypochlorous acid may be responsible for bleaching of the color GC through destruction of the extended chromophoric system.

\section{Conclusion}

The proposed methods are applicable for the assay of CTZ and have the advantage of wider range under Beer's law limits. The decreasing order of sensitivity and $\lambda_{\max }$ among the proposed methods are $\mathrm{C}>\mathrm{A}>\mathrm{B}$ and $\mathrm{C}>\mathrm{A}=\mathrm{B}$ respectively. The proposed methods are simple, selective and can be used in the routine determination of CTZ in bulk samples and formulations with reasonable precision and accuracy.

\section{References}

1. Arun K, Saravanan C, Balachandar R, Kumuthavalli M V and Jayakar B, J Chem Pharm Res., 2010, 2(1), 424-231.

2. $\quad$ Fang Ting-Ting and Sha Ou, Guangpu Shiyanshi, 2010, 27(2), 630.

3. Moreno Andreia de Haro and Salgado Herida Regina Nunes, J AOAC Int., 2009, 92(3), 820.

4. Moreno Andreia de Haro and Salgado Herida Regina Nunes, Anal Lett., 2008, 41(12), 2143.

5. Salem Hesham, Badr El-Din Khalid M,Saleh Gamal A and Kheir Afaf Aboul, Bull Fac Pharm., 2006, 44(1), 215.

6. Moreno Andreia de Haro and Salgado Herida Regina Nunes, J AOAC Int., 2009, 92(3), 820-823.

7. Mruthyunjayaswamy B H M, Hiremath Basavaraj, Malipatail S M, Raju S Appala, Asian J Chem., 2007, 19(5), 3770-3774.

8. Jane J, Subrahmanyam E V S and Sathyanarayana D, Asian J Chem., 2005, 18(1), 721. 
9. Amin A S, Khallil H M and Saleh H M, Scientia Pharmaceutica, 2001, 69(2), 143.

10. El Walily Abdel Fattah M, Abdel Kader Gazy Azza, Belal Saied F and Khamis Essam F, Spectroscopy Lett., 2000, 33(6), 931-948.

11. El-Saharty Y S, Bull Fac Pharm., 2000, 38(1), 1.

12. Raman V, Sarojini B H Geetha, Shobana A, Umarani M, Lajapathie Kavitha, Meyyanathan S N and Suresh B, Eastern Pharmacist, 2000, 43, 143.

13. Li Wei and Li Huimin, Zhongnan Yaoxue, 2010, 8, 256.

14. Shi Li-juan, Yuan Tao and Tan You-ming, Huanjing Yu Jiankang Zazhi, 2009, 26, 911.

15. Zhang Yi-yong, Ma Xiao-ling, Du Fei, Jia Heng-min, Chen Yun and Fu Guang-lin, Anhui Yiyao, 2009, 13, 24.

16. Wei Minji, Sun Lulu and Fang Liming, Yaowu Fenxi Zazhi , 2009, 29, 773.

17. Nemutlu Emirhan, Kir Sedef, Katlan Doruk and Beksac M Sinan, Talanta, 2009, 80, 117.

18. Liang Jiancheng, Wang Fangfang, Huang Yikun and Xie Ling, Zhongguo Yaoshi, 2008, 11, 1293.

19. Zhang Dong, Guo Yi, Sun Wei and Han Guojiang, Zhongguo Yaoye, 2008, 17, 34.

20. Moreno Andreia de Haro and Salgado Herida Regina Nunes, J AOAC Int., 2008, 91(4), 739.

21. Denooz Raphael and Charlier Corinne, J Chrom B, 2008, 864, 161-167.

22. Xia Dong-Ya, Guo Tao, Chen Yan and Qin Hai-Xu, Jiefangjun Yaoxue Xuebao, 2007, 23, 41.

23. Huang Li, Zhongguo Kangshengsu Zazhi., 2005, 30, 588.

24. Shi Cen, Liang Xiaojun, Chu Hong and Wu Rujin, Zhongguo Yaoxue Zazhi, 2005, 40, 72.

25. Ai Yousheng, Xu Chuhong, Chen Huating, Wang Xia and Huazhong Keji Daxue Xuebao, Yixueban , 2004, 33, 512.

26. Fang Juan-juan, Yu Wei-ping, Xing Yan-xia, Wei Kai-bin and Dongnan Daxue Xuebao, Yixueban, 2004, 23, 388.

27. Meng Xiang-ming, Meng Zhi-yun, Zhang Liang and Dou Gui-fang, J Chine Pharm Sci., 2004, 13(4), 267.

28. Chan C Y, Chan K and French G L, J Antimicrobial Chemotherapy, 1986, 18(4), 537-545.

29. Arcelloni Cinzia, Basile Monica, Vaiani Roberto, Bonini Pierangelo and Paroni Rita, J Chrom A, 1996, 742(23), 121-126. 